\title{
RISK OF JAUNDICE FOLLOWING TRANSFUSION WITH POOLED PLASMA OR SERUM
}

\author{
BY \\ LANCELOT HOGBEN, F.R.S.
}

It has been independently shown by Spurling, Shone, and Vaughan (1946), and by Truelove and Hogben (1947), that the use of pooled serum or plasma, in contradistinction to whole blood from individual donors, increases the risk of hepatitis following blood transfusion. This conclusion raises the elementary statistical problem: how does reduction of the number of contributors to a blood pool diminish the attendant risk of jaundice? The issue derives some practical interest from a radical change of procedure with respect to the number of contributors to a single pool of serum or plasma during recent years. According to information supplied to the Directorate of Hygiene (War Office) by the Ministry of Health, " small pool" material (blood from 10 donors) was issued to selected transfusion centres from June to September, 1945. The writer has not been able to elicit definite information with respect to considerations which determined the decision to adopt the number 10. The object of what follows is to elucidate the issue on the basis of such figures as are available and to clarify what information would be requisite to a precise evaluation of the risks attendant on a particular pool size.

According to information supplied by the Army Transfusion Service it was customary throughout the war for soldiers treated with pooled plasma to receive from one to two bottles. For simplicity, we may here assume that each patient received one bottle without detracting from the relevance of the ensuing argument for which the following symbols suffice:

\footnotetext{
$p$ is the proportion of donors who carry the putative virus, i.e. the incidence of the icterogenic agent in the general population;

$m$ is the proportion of persons susceptible thereto;

$P$ the risk of a manifest attack of jaundice consequent on transfusion from a blood sample to which $n$ donors contribute.
}

The probability that no one of $n$ donors will carry the icterogenic agent is $(1-p)^{\mathrm{n}}$. The probability that at least one will be a carrier is the probability that this condition will not be realized. It is therefore:

$$
1-(1-p)^{n}
$$

The probability of a manifest attack is that of two independent events: (a) that 
the blood sample contains the icterogenic agent, that is, that at least one of the donors is a carrier; (b) that the individual is susceptible. Hence we have:

$$
P=m\left(1-\overline{1-p}^{n}\right)
$$

If each patient receives $b$ bottles from the same pool the above holds good, if from different pools, we have to replace $n$ by $b n$ in (i) and (ii) becomes:

$$
P=m(1-\overline{1-p})
$$

Though we have not reliable estimates of $m$ or $p$ to determine the variation of $P$ with respect to $n$ in the above expression, we have some highly suggestive indications concerning the limits between which either $m$ or $p$ lie. Evidently $m$ must be less than (1-p) since a healthy carrier is by definition someone who is not susceptible. About 50 per cent. Army syphilitics incurred jaundice from blood distributed by imperfectly sterilized syringes before the introduction of new instructions in the fall of 1943. With due regard to the possible existence of more than one type of blood-spread icterogenic substances and to the possibility that syphilis itself increases susceptibility to such, we may provisionally infer that $m$ is at least as great as $\frac{1}{2}$, and that its true value therefore lies between 0.5 and $1 \cdot 0$. From the data of Truelove and Hogben we have an approximate value for the Army incidence (5 per cent.) of jaundice following transfusion of pooled plasma from large samples, i.e. from 500 to 600 donors, if supplied by the Army. Transfusion Service or from 300 if supplied from civilian centres. On the basis of a larger sample Spurling, Shone, and Vaughan cite an incidence of 7 per cent. Let us therefore assume that the range $m=0.25,0.50,0.90$ and the range $n b=300,500,700$ defines the limits within which a representative estimate of $p$ will lie. To evaluate $p$ subject to these conditions we may write (iii) in the form:

$$
\text { bn } \log (1-p)=\log (m-P)-\log m
$$

\begin{tabular}{|c|c|c|c|}
\hline \multirow{2}{*}{$m$} & \multicolumn{3}{|c|}{$b n$} \\
\hline & 300 & 500 & 700 \\
\hline $0 \cdot 10$ & 0.00305 & 0.00183 & 0.00131 \\
\hline 0.50 & 0.000426 & 0.000256 & $0 \cdot 000183$ \\
\hline 0.90 & 0.000229 & 0.000138 & 0.000098 \\
\hline
\end{tabular}

By taking $P$ as 0.06 (6 per cent.) we then get the values for $p$ shown in Table $I$.

TABLE I

We may now tabulate (Table II) the result of using (ii) to evaluate $P$ for different values of $n$ in accordance with the least, the median, and the greatest values of $p$ in Table $I$ and the appropriate value of $m$ corresponding to each such estimate.

On the basis of the tentative figures used for the estimates given above, it appears that reduction of the size of the pool from 500 to 10 entails a very substantial 
reduction of the risk of infection; but this conclusion is by no means obvious. Its validity depends on the values assigned to the incidence of the icterogenic virus in the general population. The foregoing calculations assume a value for $m$ suggested by the incidence of jaundice in the syphilitic population. This is open

TABLE II

\begin{tabular}{|c|c|c|c|c|c|c|c|c|c|}
\hline \multirow{2}{*}{$p$} & \multicolumn{9}{|c|}{$b n$} \\
\hline & 1 & 3 & 5 & 9 & 10 & 25 & 100 & 200 & 500 \\
\hline 0.000098 & 0.000088 & 0.00027 & 0.00045 & 0.00080 & 0.00088 & 0.00222 & 0.0087 & 0.0174 & 0.044 \\
\hline 0.000256 & $0 \cdot 000128$ & 0.00038 & 0.00064 & 0.00115 & 0.00127 & 0.00319 & 0.0126 & 0.0252 & 0.060 \\
\hline 0.00305 & 0.000305 & 0.000912 & 0.00152 & 0.00271 & 0.00301 & 0.00760 & 0.0271 & 0.0451 & 0.078 \\
\hline
\end{tabular}

to criticism that syphilis may itself be propitious to the pathogenic action of the virus; and there is indeed some evidence (Truelove and Hogben, 1947) to support this surmise. Among Army patients who received transfusion of whole blood from single donors, the incidence of jaundice was 0.81 per cent. If we take this figure at its face value we may put

$$
\begin{gathered}
m p=0.0081 \\
\text { and } \\
m\left(1-\overline{1-p}^{b n}\right)=0.05
\end{gathered}
$$

If we take $b n$ as 500 , we derive $0 \cdot 16$ as our estimate of $p$. On the basis of the value we obtain the following estimate of the relative risks attendant on transfusion from pools from $1,5,10,25$, and 100 donors:

\begin{tabular}{c|c|c|c|c}
\hline 1 & 5 & 10 & 25 & 100 \\
\hline 1.0 & $3 \cdot 6$ & $5 \cdot 0$ & $6 \cdot 2$ & $6 \cdot 25$ \\
\hline
\end{tabular}

The statistical error on the value assigned to $m p$ in this context is very large. Spurling, Shone, and Vaughan did not in fact record any case of jaundice in a larger sample of patients who received blood from individual donors. It would therefore be unjustifiable to cite the figures of the last table as evidence for the view that the reduction of donors contributing to a pool from 500 to 10 does not conspicuously reduce the attendant risk of jaundice. What such figures do serve to show is the need for more exact statistics than those at present available, as a rational basis for a decision of the sort under consideration.

\section{REFERENCES}

Spurling, N., Shone, J., and Vaughan, J. (1946). Brit. med. J., 2, 409.

Truelove, S. C., and Hogben, L. (1947). Brit. J. soc. Med., 1, 18. 